Western University

Scholarship@Western

$12-13-2014$

\title{
Registration of in-vivo to ex-vivo MRI of surgically resected specimens: A pipeline for histology to in-vivo registration.
}

\author{
Maged Goubran \\ Western University, mgoubran@uwo.ca \\ Sandrine de Ribaupierre \\ Western University, sderibau@uwo.ca \\ Robert R Hammond \\ Western University, robert.hammond@lhsc.on.ca \\ Catherine Currie \\ Western University, ccurrie@uwo.ca \\ Jorge G Burneo \\ Western University, jburneo2@uwo.ca
}

See next page for additional authors

Follow this and additional works at: https://ir.lib.uwo.ca/biophysicspub

Part of the Medical Biophysics Commons

\section{Citation of this paper:}

Goubran, Maged; de Ribaupierre, Sandrine; Hammond, Robert R; Currie, Catherine; Burneo, Jorge G; Parrent, Andrew G; Peters, Terry M; and Khan, Ali R, "Registration of in-vivo to ex-vivo MRI of surgically resected specimens: A pipeline for histology to in-vivo registration." (2014). Medical Biophysics Publications. 22.

https://ir.lib.uwo.ca/biophysicspub/22 


\section{Authors}

Maged Goubran, Sandrine de Ribaupierre, Robert R Hammond, Catherine Currie, Jorge G Burneo, Andrew G Parrent, Terry M Peters, and Ali R Khan 


\title{
Registration of in-vivo to ex-vivo MRI of surgically resected specimens: A pipeline for histology to in-vivo registration
}

\author{
Maged Goubran ${ }^{\mathrm{a}, \mathrm{c}, *}$, Sandrine de Ribaupierre ${ }^{\mathrm{b}, \mathrm{c}}$, Robert R. Hammond ${ }^{\mathrm{d}}$, Catherine Currie ${ }^{\mathrm{a}}$, \\ Jorge G. Burneo ${ }^{\mathrm{b}}$, Andrew G. Parrent ${ }^{\mathrm{b}}$, Terry M. Peters ${ }^{\mathrm{a}, \mathrm{c}, \mathrm{e}}$, Ali R. Khan ${ }^{\mathrm{a}, \mathrm{e}}$ \\ a Imaging Research Laboratories, Robarts Research Institute, London, Ontario, Canada \\ ${ }^{\mathrm{b}}$ Epilepsy Program, Department of Clinical Neurological Sciences, Western University \& London Health Sciences Centre, London, Ontario, Canada \\ ${ }^{\mathrm{c}}$ Biomedical Engineering, Western University, London, Ontario, Canada \\ d Department of Pathology, Division of Neuropathology, Western University E London Health Sciences Centre, London, Ontario, Canada \\ e Department of Medical Biophysics, Western University, London, Ontario, Canada
}

\section{H I G H L I G H T S}

- We present a protocol for registration of in-vivo to ex-vivo brain specimens.

- This protocol completes a registration pipeline for histology to in-vivo MRI.

- A TRE of $1.35 \pm 0.11 \mathrm{~mm}$ (neocortex) and $1.41 \pm 0.33 \mathrm{~mm}$ (hippocampus) was found.

- Deformable registration significantly improved the registration accuracy.

- This pipeline allows for the assessment of pathological correlates in MRI.

\section{A R T I C L E I N F O}

\section{Article history:}

Received 24 June 2014

Received in revised form 3 December 2014

Accepted 6 December 2014

Available online 13 December 2014

\section{Keywords:}

Epilepsy

MRI

Histology

Image registration

Pathology

Anterior temporal lobectomy

\begin{abstract}
A B S T R A C T
Background: Advances in MRI have the potential to improve surgical treatment of epilepsy through improved identification and delineation of lesions. However, validation is currently needed to investigate histopathological correlates of these new imaging techniques. The purpose of this work is to develop and evaluate a protocol for deformable image registration of in-vivo to ex-vivo resected brain specimen MRI. This protocol, in conjunction with our previous work on ex-vivo to histology registration, completes a registration pipeline for histology to in-vivo MRI, enabling voxel-based validation of novel and existing MRI techniques with histopathology.

New method: A combination of image-based and landmark-based 3D registration was used to register in-vivo MRI and the ex-vivo MRI from patients $(N=10)$ undergoing epilepsy surgery. Target registration error (TRE) was used to assess accuracy and the added benefit of deformable registration.

Results: A mean TRE of $1.35 \pm 0.11$ and $1.41 \pm 0.33 \mathrm{~mm}$ was found for neocortical and hippocampal specimens respectively. Statistical analysis confirmed that the deformable registration significantly improved the registration accuracy for both specimens.

Comparison with existing methods: Image registration of surgically resected brain specimens is a unique application which presents numerous technical challenges and that have not been fully addressed in previous literature. Our computed TRE are comparable to previous attempts tackling similar applications, as registering in-vivo MRI to whole brain or serial histology.

Conclusion: The presented registration pipeline finds dense and accurate spatial correspondence between in-vivo MRI and histology and allows for the spatially local and quantitative assessment of pathological correlates in MRI.
\end{abstract}

(c) 2014 Elsevier B.V. All rights reserved.

\footnotetext{
* Corresponding author at: PO Box 5015, 1151 Richmond St. North, London, Ontario N6A 5B7, Canada. Tel.: +1 5197196705.

E-mail address: mgoubran@robarts.ca (M. Goubran).
}

\section{Introduction}

Approximately $30 \%$ of all patients with epilepsy are considered medically intractable, that is about one third of patients do not achieve remission with antiepileptic drugs (Engel, 1998). Surgical excision of the affected brain region is an effective treatment 
for drug-resistant focal epilepsy (Engel et al., 1992), with a recent long-term clinical study of surgical outcomes reporting that fewer than $60 \%$ of patients remained seizure-free at 10 years follow up (de Tisi et al., 2011). Such data have motivated the need for better pre-operative imaging and image analysis techniques to locate the epileptogenic foci and disease-related pathological tissue more accurately and provide better surgical planning. Current clinical magnetic resonance imaging (MRI) protocols lack sensitivity, as more than $30 \%$ of patients have no evidence of brain lesions (Sylaja et al., 2004). Quantitative techniques such as diffusion tensor imaging (DTI), relaxometry mapping, voxel-based morphometry, and cortical thickness analysis have demonstrated increased sensitivity in lesion detection over routine or clinical MRI protocols (Bernasconi et al., 2000, 2004; Bernhardt et al., 2009). These techniques have the potential to better delineate the epileptogenic zone and thus improve surgical outcomes, however, validation is currently needed to investigate and describe histopathological correlates of these imaging techniques (Eriksson et al., 2007; Howe et al., 2010). In order to carry out this validation effectively, accurate registration must be performed to obtain a dense spatial correspondence between in-vivo MR images and histology images of surgical specimens.

MRI to histology registration is far from trivial due to the significant deformations undergone by the brain tissue during surgery, handling, and histological processing. These deformations can be split into two main categories, those occurring during surgical resection and those during histological processing (Dauguet et al., 2007). Those due to surgical resection are three dimensional mechanical deformations that take place once brain tissue is resected, due to its tendency to deform when separated from neighbouring tissue. The histological processing deformations are three dimensional, occurring during sectioning or due to non uniform shrinkage induced by formalin fixation, as well as two dimensional (within-slice) distortions due to stretching of microtome cut sections on a water bath, spreading histology slices over glass slides and staining. The deformations induced during histological processing can be isolated from those from surgery and handling by employing an intermediary MRI image of the specimen or using blockface images for histological reconstruction, splitting the in-vivo MRI to histology registration procedure into two distinct problems (in-vivo to reference and reference to histology). As described in our previous work registering ex-vivo MRI to sparsely sectioned hippocampal and neocortical temporal lobe specimens, the intermediate ex-vivo MRI or blockface stack can function as an anatomical reference with which the 2D histological slices can be corrected against (Goubran et al., 2013). In this work, however, we focus on the first problem of registering the in-vivo MRI to the intermediate ex-vivo MRI, and completing a pipeline for histology to in-vivo MRI registration in temporal lobe epilepsy.

There have been many attempts in the literature to register invivo MR images of many organs, such as the prostate (Ward et al., 2010; Chappelow et al., 2011), to histology slices. Extrapolating these registration techniques to the brain may not be practical since the brain has very different biomechanical properties than other organs and is prone to deformation. Moreover, algorithms optimized for registering other resected organs generally do not deal with part-to-whole registration, and thus may not be applicable in our problem. In the past two decades, there have also been many studies specifically dealing with in-vivo brain MRI to post-mortem histology. The majority of these studies focused on primates (Dauguet et al., 2007; Malandain et al., 2004; Breen et al., 2005; Ceritoglu et al., 2010; Choe et al., 2011) or rodents (Jacobs et al., 1999; Humm et al., 2003; Meyer et al., 2006; Lebenberg et al., 2010; Yang et al., 2012; Liu et al., 2012). The few studies that registered human brain MRIto histology were performed on wholebrain (Schormann et al., 1995; Kim et al., 2000; Singh et al., 2008), or single hemisphere (Yelnik et al., 2007; Osechinskiy and Kruggel, 2011) post-mortem serially sectioned data (Amunts et al., 2013) created a 3D model of single subject's brain using post-mortem histological sections reconstructed at $20 \mu \mathrm{m}$ isotropic resolution and registered it to a $\mathrm{T} 1$ average atlas created from 24 subjects. Eriksson et al. (2005) reported registering histology of neocortical specimens from anterior temporal lobectomies to in-vivo MRI; however, their approach only involved visually selecting the closest coronal MRI slice for each histology slide, and did not attempt to find a dense correspondence between each histology slide and its corresponding MRI slice.

This study focuses on finding correspondences between in-vivo and ex-vivo MRI, which enables the validation of in-vivo imaging findings using higher-resolution ex-vivo scans. It also bridges information from histology to ex-vivo data and finally to the clinically relevant pre-operative images when combining our previous work with the current study. Image registration of a deformed cut specimen to the original brain, that is part-to-whole registration, is challenging because similarities between the images have been constrained to a meaningful sub-region of the in-vivo image that is variable from specimen to the other (due to different resection strategies and substantially variable specimen shapes and volumes). The presented registration approach for this problem employs an automated initialization as well as a landmark-based rigid registration, followed by a landmark deformable registration for hippocampal specimens and an image-based non-rigid warping for neocortical specimens. Using anatomical landmarks is a reliable technique for registration that exploits the operator's anatomical expertise and enforces registration constraints based on the placed landmarks.

\section{Methods}

\subsection{Recruitment, surgery and specimen acquisition}

Temporal lobe epilepsy patients who were candidates for anterior temporal lobectomy (ATL) surgery were recruited for this study. Patients had preoperative investigations including neuropsychological testing and $1.5 \mathrm{~T}$ clinical MRI scans which included T1w, T2w, FLAIR, and diffusion-weighted sequences. Patients were monitored with scalp-based electroencephalogram (EEG) video telemetry for seizure characterization, with three patients requiring subdural electrodes placement. In addition to the $1.5 \mathrm{~T}$ clinical MRI scans performed at the hospital, patients underwent a series of scans on 3T and 7T MRI research scanners, described in the in-vivo MRI subsection. Our study cohort included 10 temporal lobe patients who underwent epilepsy surgery and the resection of two specimens, temporal lobe neocortex and hippocampus, as part of an ongoing project at the Robarts Research Institute. Two hippocampal specimens were not obtained en-bloc due to the use of the cavitron ultrasonic surgical aspirator (CUSA) device during surgery, and were thus excluded from this study. This project was approved by the office of research and ethics of Western University, and informed consent was obtained from all patients prior to their recruitment in the study. Table 1 summarizes the age, gender, onset age, seizure origin as well as clinical MRI and pathology findings for our patient cohort.

\subsection{Patient in-vivo MR Imaging and maps generation}

All patients underwent pre-operative imaging on a $3 \mathrm{~T}$ Discovery MR750 scanner (General Electric, Milwaukee, WI, U.S.A.) with a 32 channel head coil and consisted of relaxation mapping, diffusion-tensor imaging and resting-state functional imaging. For T1 mapping the 'DESPOT1-HIFI' approach (Deoni, 2007) 
Table 1

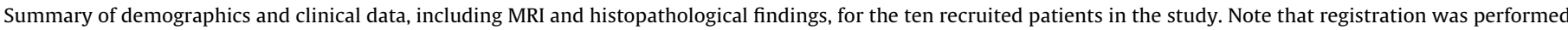
on both hippocampus and neocortex specimens for eight patients (neocortex only for cases 1 and 4).

\begin{tabular}{|c|c|c|c|c|c|c|c|c|}
\hline \multirow[t]{2}{*}{ Patient } & \multirow[t]{2}{*}{ Gender } & \multirow[t]{2}{*}{ Age } & \multirow[t]{2}{*}{ Age of onset } & \multirow[t]{2}{*}{ Seizure origin } & \multirow[t]{2}{*}{ MRI } & \multicolumn{2}{|l|}{ Pathology } & \multirow[t]{2}{*}{ Ex-vivo MRI } \\
\hline & & & & & & Hippocampus & Neocortex & \\
\hline 1 & $\mathrm{~F}$ & 26 & 19 & Right & TS & Gliosis & TS & $3 \mathrm{~T}$ \\
\hline 2 & $\mathrm{~F}$ & 25 & 17 & Left & Prev. resection & Gliosis & Gliosis, atypical cells in WM & $3 \mathrm{~T}$ \\
\hline 3 & M & 20 & 3 & Left & LMTS & MTS & Gliosis, MAA & $3 \mathrm{~T}$ \\
\hline 4 & M & 18 & 14 & Right & Possible RMTS & Gliosis $^{\mathrm{a}}$ & Gliosis, MAA & $3 \mathrm{~T}$ \\
\hline 5 & M & 48 & 36 & Left & LMTS & MTS & Gliosis & $3 \mathrm{~T}$ \\
\hline 6 & $\mathrm{~F}$ & 50 & 47 & Left & GM/WM blurring & Gliosis $^{\mathrm{a}}$ & Gliosis & $3 \mathrm{~T}$ \\
\hline 7 & $\mathrm{~F}$ & 31 & 28 & Right & Normal & Negligible gliosis & Mild gliosis, MAA & $9.4 \mathrm{~T}$ \\
\hline 8 & M & 19 & 5 & Left & LMTS & MTS & Gliosis & $9.4 \mathrm{~T}$ \\
\hline 9 & $\mathrm{~F}$ & 43 & 3 & Right & RMTS & MTS & Gliosis & $9.4 \mathrm{~T}$ \\
\hline 10 & M & 34 & 15 & Left & LMTS & MTS & Gliosis, focal MAA & $9.4 \mathrm{~T}$ \\
\hline
\end{tabular}

a Pathology did not contain sufficient tissue for diagnosis. MTS: mesial temporal sclerosis, TS: tuberous sclerosis, MAA: mild architectural abnormalities.

was used and involved acquisition of two 3D SPGR sagittal T1-weighted images $\left(\mathrm{TR}=8.36 \mathrm{~ms}, \mathrm{TE}=3.712 \mathrm{~ms}\right.$, flip angles $=4^{\circ}$ and $18^{\circ}$, matrix $=220 \times 220$, slice thickness $=1$, FOV $=220 \mathrm{~mm}$ ), as well as an additional inversion-prepared SPGR for B1 mapping $\left(\mathrm{TR}=6.46 \mathrm{~ms}, \mathrm{TE}=3.1 \mathrm{~ms}\right.$, flip angle $=5^{\circ}$, matrix $=220 \times 128$, slice thickness $=0.5$, FOV $=220 \mathrm{~mm}$ ). For T2 mapping the 'DESPOT2FM' approach (Deoni, 2009), whereby five balanced steady-state free precession (bSSFP) images were acquired with flip angles $5^{\circ}, 35^{\circ}$ and $68^{\circ}$ with phase cycling patterns $\theta \mathrm{RF}=0^{\circ}$ and $180^{\circ}(\mathrm{TR}=4.608 \mathrm{~ms}, \mathrm{TE}=2.356 \mathrm{~ms}$, matrix $=220 \times 220$, slice thickness $=1, \mathrm{FOV}=220 \mathrm{~mm}$ ). The $\mathrm{T} 1 \mathrm{map}$ was subsequently used for the registration protocol for all the patients. Diffusion tensor imaging (DTI), though not used to guide the registration, was performed using an axial spin-echo echo-planar imaging (EPI) sequence with 41 diffusion directions and a b-value of 1000 (TR=1100 ms, $\mathrm{TE}=63.2 \mathrm{~ms}$, flip angle $=90^{\circ}$, matrix $=96 \times 96$, slice thickness $=2.5$, $\mathrm{FOV}=240 \mathrm{~mm}$ ). Fractional anisotropy (FA) and mean diffusivity (MD) maps were computed after tensor estimation from the DTI data using the FDT tool of the FSL image analysis suite.

Patients also underwent high resolution structural imaging at $7 \mathrm{~T}$ (Agilent Technologies, Santa Clara, CA, U.S.A./Siemens, Erlangen, Germany), and are also shown in conjunction with the histology for a qualitative comparison. The $7 \mathrm{~T}$ imaging protocol comprised a T1-weighted magnetization prepared rapid gradient echo (MPRAGE) sequence with a $0.75 \mathrm{~mm}$ isotropic resolution $\left(\mathrm{TR}=8.42 \mathrm{~ms}, \mathrm{TE}=3.9 \mathrm{~ms}\right.$, flip angles $=11^{\circ}$, matrix $=220 \times 294 \times 230, \mathrm{FOV}=150 \times 220 \times 172 \mathrm{~mm})$ and a T2-w turbo spin echo (TSE) sequence with a $0.6 \mathrm{~mm}$ isotropic resolution $(\mathrm{TR}=3750 \mathrm{~ms}$, Effective $\mathrm{TE}=470.83 \mathrm{~ms}$, Echo spacing $=4.73 \mathrm{~ms}$, flip angles $=90^{\circ}$, matrix $=260 \times 366 \times 266, \mathrm{FOV}=156 \times 220 \times 160 \mathrm{~mm}$, echo train length $\left.(\mathrm{ETL})=199, n_{\mathrm{avg}}=4\right)$.

\subsection{Specimen ex-vivo MR imaging}

Following surgery, the resected tissue specimens were transferred to the Robarts Research Institute for ex-vivo specimen imaging followed by processing in the pathology lab at the hospital. After resection, each specimen was oriented by the operating neurosurgeon, photographed and transported on ice to the imaging lab. The ex-vivo scanning was performed after overnight fixation in $10 \%$ formalin. Each specimen was wrapped in gauze for stabilization, transferred to suitably sized containers for imaging, and immersed in a fluorine-based lubricant 'Christo-lube MCG 1046' (Lubrication Technology, Inc) prior to imaging to avoid susceptibility artifacts at the tissue boundaries. The specimen scanning was performed on the $3 \mathrm{~T}$ scanner used for patient imaging with both hippocampal and neocortical specimens imaged in the same field of view using a 6 channel coil designed to image the carotid artery. T2-weighted fast imaging employing steady state acquisition (FIESTA) images $(\mathrm{TR}=8.17 \mathrm{~ms}, \quad \mathrm{TE}=4.08 \mathrm{~ms}$, flip angle $=40^{\circ}, \quad N=2, \quad$ matrix $=200 \times 200$, slice thickness $=0.4$, FOV $=70 \mathrm{~mm}$ ) with a resolution of $0.35 \times 0.35 \times 0.4 \mathrm{~mm}$, as well as fast gradient echo (fastGRE) scans with sixteen echoes $\left(\mathrm{TR}=65.0 \mathrm{~ms}, \mathrm{TE}=38.9 \mathrm{~ms}\right.$, flip angle $=40^{\circ}$, matrix $=200 \times 200$, slice thickness $=0.4, F O V=70 \mathrm{~mm}$ ) were acquired for the study, for a total scan time of less than $2 \mathrm{~h}$.

For cases where overnight imaging was feasible and not disruptive to the clinical workflow $(N=4)$, scanning was performed on a $9.4 \mathrm{~T}$ small bore Varian MR magnet (Varian, Palo Alto, CA, U.S.A.) for improved image resolution and signal-to-noise (SNR), as an alternative to the $3 \mathrm{~T}$ scan. Each specimen was imaged separately using different coils for a total time of $16 \mathrm{~h}$ per specimen. The hippocampal specimens were imaged in a millipede birdcage MP30 coil (Agilent, Santa Clara, CA, U.S.A.) and the neocortical specimens were scanned with an in-house developed coil. True fast imaging with steady state precession (TrueFisp) images $\left(\mathrm{TR}=7.6 \mathrm{~ms}, \mathrm{TE}=3.8 \mathrm{~ms}\right.$, flip angle $\left.=30^{\circ}\right)$ were acquired with a resolution of $0.1 \mathrm{~mm}$ isotropic, a FOV of $38 \times 25.6 \times 19.2 \mathrm{~mm}$; and a resolution of $0.2 \mathrm{~mm}$ isotropic (FOV of $50 \times 26 \times 44$ ), for hippocampal and neocortical specimens respectively. Spin-echo diffusion sequences were also acquired $(\mathrm{TR}=7.6 \mathrm{~ms}$, $\mathrm{TE}=3.8 \mathrm{~ms}$, slice thickness $=0.4 \mathrm{~mm}$ ) with an in-plane resolution of $0.1 \times 0.1 \mathrm{~mm}$ and FOV of $38 . \times 25.6 \mathrm{~mm}$, and an in-plane resolution of $0.2 \times 0.2 \mathrm{~mm}$ and FOV of $50 \times 26$, for hippocampal and neocortical specimens respectively. The T2-weighted images from either protocol were used in the registration pipeline.

\subsection{Histological processing}

Following ex-vivo imaging, the specimens were accessioned and grossed at the Department of Pathology at the University Hospital of London Health Sciences Centre. They were then cut midway, anterior-posterior and each half of the specimen was embedded in agar for a stabilization effect during slicing. Each half was then sectioned into $4.4 \mathrm{~mm}$ apart coronal blocks using a deli slicer (Globe Food Equipment Company, Dayton, OH, U.S.A.). The median number of $\mathrm{Hp}$ and Neo blocks was 8 and 12 respectively. Each block was embedded in paraffin and mounted on a microtome where $8 \mu \mathrm{m}$ thick sections were cut from the face of each block and mounted on slides. One slide from each block was stained with hematoxylin and eosin (H\&E) and select blocks processed for immunohistochemistry (IHC), mainly glial fibrillary acidic protein (GFAP) (rabbit plolyclonal antibody; 1:4000; Dako, Agilent Technologies, Santa Clara, CA, U.S.A.) and neuronal nuclei (NeuN) (mouse monoclonal antibody; 1:400; EMD Millipore, Billerica, Massachusetts) as determined on clinical grounds by the neuropathologist on duty.

For the hippocampus, on average about a quarter of the blocks were additionally stained with GFAP. As for the neocortex, about 
half of the blocks were additionally stained with both IHC stains. GFAP was incubated for a total of 50 min while NeuN was incubated for $65 \mathrm{~min}$ and retrieval for both IHC stains was performed at $\mathrm{pH}$ 9.0. Batch IHC processing was performed on a Dako Autostainer Link 48 (Dako, Agilent Technologies, Santa Clara, CA, U.S.A.) to minimize variability between slides. The resulting slides were digitized on a ScanScope GL (Aperio Technologies, Vista, CA, U.S.A.) bright field slide scanning system at a maximum of $20 \times$ optical zoom, and stitched to form full-frame multi-resolution images stored in BigTIFF file format (maximum pixel resolution $0.5 \mu \mathrm{m}$ ).

\subsection{Image registration}

The goal of the image registration for a given specimen is to find correspondence between the $3 \mathrm{D}$ in-vivo MRI, denoted as $I_{i n}$, and the set of $N 2 \mathrm{D}$ histology slides, denoted as $\left\{I_{h 2 d}^{i}\right\} i=1 \ldots N$. We divide this process into two distinct steps through the use of an intermediate 3D ex-vivo MRI of the specimen, $I_{e x}$. Our previous work (Goubran et al., 2013) described and validated methodology for generating a 3D reconstruction of the histology slides, $I_{h 3 D}$, with an iterative approach that alternates between aligning $I_{e x}$ to $I_{h 3 D}$, and correcting for within-slices deformations using the aligned $I_{e x}$ as a reference. The registration detailed in this work relates to finding the transformations between $I_{i n}$ and $I_{e x}$, thus connecting images obtained in-vivo with histological slides, as depicted in Fig. 1. These transformations are obtained in a hierarchical fashion, beginning with an initial translation, $T_{e x, i n}^{\text {trans }}$, a landmark-based similarity transformation, $T_{e x, i n}^{\operatorname{sim}}$, and finally a non-rigid deformation, $\Phi_{e x, i n}$. To avoid oblique resampling of the highly anisotropic histology images for the purposes of visualization and analysis, the in-vivo and exvivo images are ultimately transformed to the space of the $3 \mathrm{D}$ reconstructed histology, Hist $3 D$, where the reconstructed coronal histology slides are stacked parallel to the anterior-posterior axis.

\subsubsection{Initialization}

Prior to image registration, the images underwent a series of pre-processing steps, carried out with command-line tools from the FSL image analysis suite (FSL, http://fsl.fmrib.ox.ac.uk) and scripts written in MATLAB (The MathWorks Inc., Natick, MA, U.S.A.). The goal of the first transformation obtained between $I_{e x}$ and $I_{i n}$, as depicted in Fig. 1, is to translate the resected specimen into in-vivo space, in close proximity to the relevant anatomical structures in the anterior temporal lobe. We performed this step in an automated fashion by computing a translation matrix, $T_{e x, i n}^{\text {trans }}$ between standardized coordinates in $I_{\text {in }}$ (anterior temporal lobe, hippocampus) and the center of mass in a foreground mask obtained from $I_{e x}$, generating the ex-vivo image $I_{e x}^{\text {trans }}$. The in-vivo coordinates were found through linear registration to the MNI152 space, where the center of the anterior temporal lobe and hippocampus in each respective hemisphere were labeled with MNI152 coordinates of $(25,-14$, $-19)$ and $(40,-2,-30)$ for the right hippocampus and temporal lobe respectively (left side coordinates had the $x$ negated). The images, $I_{e x}^{\text {trans }}$ and $I_{i n}$, were then resampled to an isotropic resolution of $0.4 \mathrm{~mm}$, and both cropped to the extents of $I_{e x}^{\text {trans }}$. This initial translation, along with the pose correction, allows for visualization of the ex-vivo and in-vivo images in the same space and greatly facilitates placement of landmarks for the following step.

\subsubsection{Landmark-based similarity transformation}

After the initial translation, alignment of the images was further refined using landmark-based registration. Landmarks were placed on the initialized ex-vivo and in-vivo images $I_{e x}^{\text {trans }}$ and $I_{i n}$ in the open source software 3D Slicer, http://www.slicer.org, version 4.2.1. For the hippocampal specimens, on average up to 10 anatomical landmarks were manually chosen on orthogonal image planes within the hippocampus proper and the hippocampal subfields, specifically the dentate gyrus, with the guidance of the hippocampal sulcus, as well as the parahippocampal gyrus depending on the extents of the resection. For the neocortical specimens, the anatomical landmarks were chosen on the surface of both the in-vivo brain and ex-vivo specimen using 3D surface models and photographs from pathological grossing of the specimen. A surfacebased approach was chosen since the rich set of cortical surface features visible on both images was amenable to localizationof corresponding landmarks. Surface visualization of the in-vivo brain was accomplished with volume-rendering of the skull-stripped and bias-corrected T1-weighted SPGR image (flip angle $18^{\circ}$ ). An average of 15 landmarks were placed on the surface of each neocortical specimen. Figs. 2 and 3 demonstrate an example of corresponding anatomical landmarks on both the in-vivo T1 map and the ex-vivo hippocampi and neocortices, and their distribution within each specimen, respectively. After placement of landmarks the optimal transformation, $T_{e x, i n}^{s i m}$ ( 7 dof rigid transform plus scaling), aligning the ex-vivo to the in-vivo set was found. The scale factor was employed to allow more flexibility than a rigid transformation to account for physical contraction of the tissue, while restricting unrealistic shearing that would be present in an affine transformation. The similarity transformation was applied to the translated ex-vivo images $I_{e x}^{\text {trans }}$ to produce, $I_{e x}^{\text {sim }}$.

\subsubsection{Non-rigid registration: hippocampus}

A non-rigid transformation, $\Phi_{e x, i n}$, is required to account for deformations of the hippocampi during and after surgery. Subsequent to the landmark-based similarity transform, we performed a deformable landmark-based registration for our hippocampal specimens using radial basis functions (RBF) for local corrections as implemented in the Plasti match plug-in (Pinter et al., 2012) in 3D Slicer. Two new sets of corresponding landmarks were placed, on $I_{e x}^{s i m}$ and $I_{i n}$, in coronal MR slices to match the coronally slice histology. After testing a range of parameters and visual analysis of the results, we performed the deformable landmark-based registration using a Gaussian RBF radius of $50 \mathrm{~mm}$ and a regularization weight of 0.1 . In some cases the regularization parameter was increased, by 0.1 increments, to ensure smoothness of the resulting deformation in regions where errors in landmark correspondence might warp the images inappropriately. An average of 12 landmarks were used per specimen to perform the registration. Since the Hist3D space was chosen as the target space for bringing in-vivo MRI and histology into alignment, the inverse deformation field $\Phi_{e x, i n}^{-1}$ was computed using the Inverse warp tool in FSL. This inverse warp was afterwards composed with the inverse transforms, $T_{e x, i n}^{\operatorname{sim}}$ and $T_{e x, i n}^{\text {trans }}$, generated in the previous sections, as well as $T_{e x, h 3 D}^{r i g}$ to bring the in-vivo image, $I_{i n}$, to the Hist3D space, as depicted in Fig. 1. The resulting composite deformation was applied to the T2 quantitative map, the FA and MD diffusion maps and structural images acquired at $7 \mathrm{~T}$ as well as clinical $1.5 \mathrm{~T}$ scans, if available, to warp them to the Hist3D space for visualization and analysis.

\subsubsection{Non-rigid registration: neocortex}

Since the neocortex specimens are larger in size, deform more regularly, and contain many salient features in the cortical folding patterns, a more automated image-based approach was chosen to provide the analogous non-rigid deformation. We performed a fast non-rigid registration on the rigidly aligned images, $I_{e x}^{\text {sim }}$ and $I_{i n}$, that makes use of a B-spline deformation field, and a normalized mutual information (NMI) cost-function (Rueckert et al., 1999; Modat et al., 2010) (NiftyReg, http://sourceforge.net/projects/niftyreg/). The algorithm estimates the transformation, $\Phi_{e x, i n}$, that maps the ex-vivo image to the in-vivo images such that the NMI cost function is minimized, with a regularization term based on the bending 


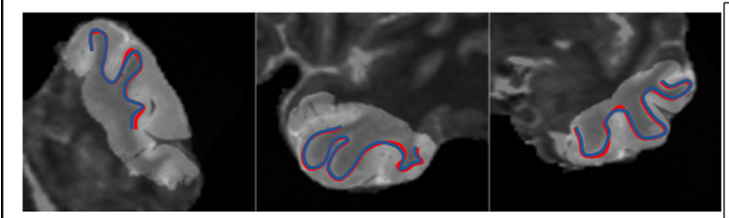

In-vivo to Ex-vivo Registration

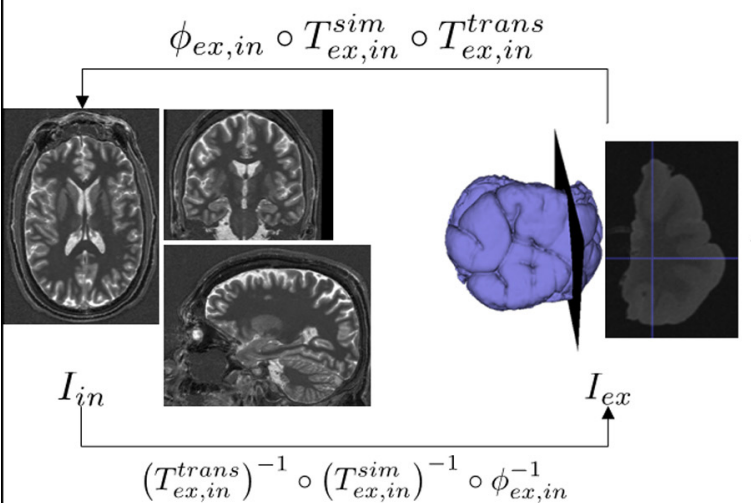

In-vivo to Histology Registration

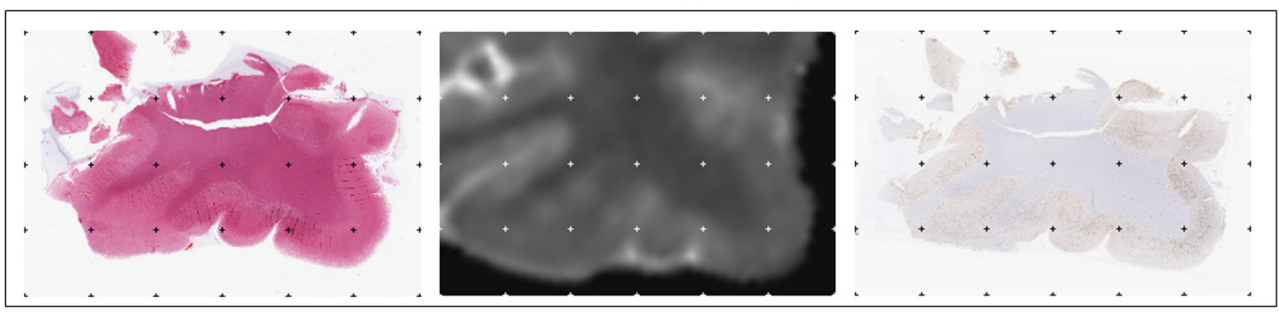

Fig. 1. In-vivo MRI to histology registration scheme depicting the transformations obtained through each registration step and the resulting images. energy at each control point. A symmetric implementation was employed that also generates an inverse warp, $\Phi_{e x, i n}^{-1}$, for warping images in the opposite direction. The B-spline registration employed a three-level multi-resolution image pyramid with final control point spacing of 7 voxels or $2.8 \mathrm{~mm}$, small enough to account for local deformations encountered and sufficiently large to avoid noise and provide a smooth deformation. We set the deformation penalty term (bending energy of the spline at a control point) as $1 \mathrm{e}^{-4}$. As employed for the hippocampal specimens, a composite deformation field, generated by combining $\Phi_{e x, \text { in }}$ and the transformations described in previous sections, was applied to our $3 \mathrm{~T}$ quantitative maps, the $7 \mathrm{~T}$ high resolution anatomical scans, if available and clinical $1.5 \mathrm{~T}$ scans to warp them to the Hist $3 D$ space.

\subsection{In-vivo MRI to histology registration}

Transformations obtained via methodology outlined in our previous study (Goubran et al., 2013) were used to reconstruct a 3D histology stack and register the ex-vivo MRI to this stack. This work also described a supplementary co-registration to bring additional immunohistochemistry (IHC) stains, such as neuronal nuclei (NeuN) and glial fibrillary acidic protein (GFAP), into alignment with the H\&E stains used for registration with the MRI. We reconstructed three dimensional RGB histology volumes from downsampled two dimensional sections $(20 \mu \mathrm{m}$ and $100 \mu \mathrm{m})$ and warped them using the previously described deformation fields to the intermediate Hist3D space.

\subsection{Registration validation and statistical analysis}

Validation of our registration protocol was achieved by computing target registration error (TRE) based on manually identified corresponding intrinsic, anatomical, landmarks on in-vivo and exvivo MR images. A set of corresponding landmarks, different from those used in the registration steps, was employed to validate our target registration accuracy to assess TRE at both the rigid and deformable stages of the protocol. All validation landmarks were placed on the MR images in their original space. To assess significant differences between the rigid and deformable registrations, we computed a Wilcoxon matched-pairs signed-rank test with a two-tailed $\alpha$ value of 0.05 between the mean TRE values of both registrations, as it does not assume a normal distribution nor dependency between the randomly selected pairs. Statistical analyses were performed in Prism 5.04 (GraphPad Software, San Diego, CA, U.S.A.).

We also assessed the sensitivity of our registration protocol to variability in landmark placement, by performing the registration on three hippocampal specimens with two independent landmark sets. The two resulting transformations for each specimen were applied to a single set of validation landmarks to assess the differences in TRE. There is also human error associated with placement of the landmarks used for validation, defined as target localization error (TLE), and this error effectively contributes to the TRE. To quantify TLE we evaluated the reproducibility of landmark placement on the in-vivo MRI given a single set of ex-vivo landmarks. TLE was calculated as an unbiased estimator of the standard deviation 


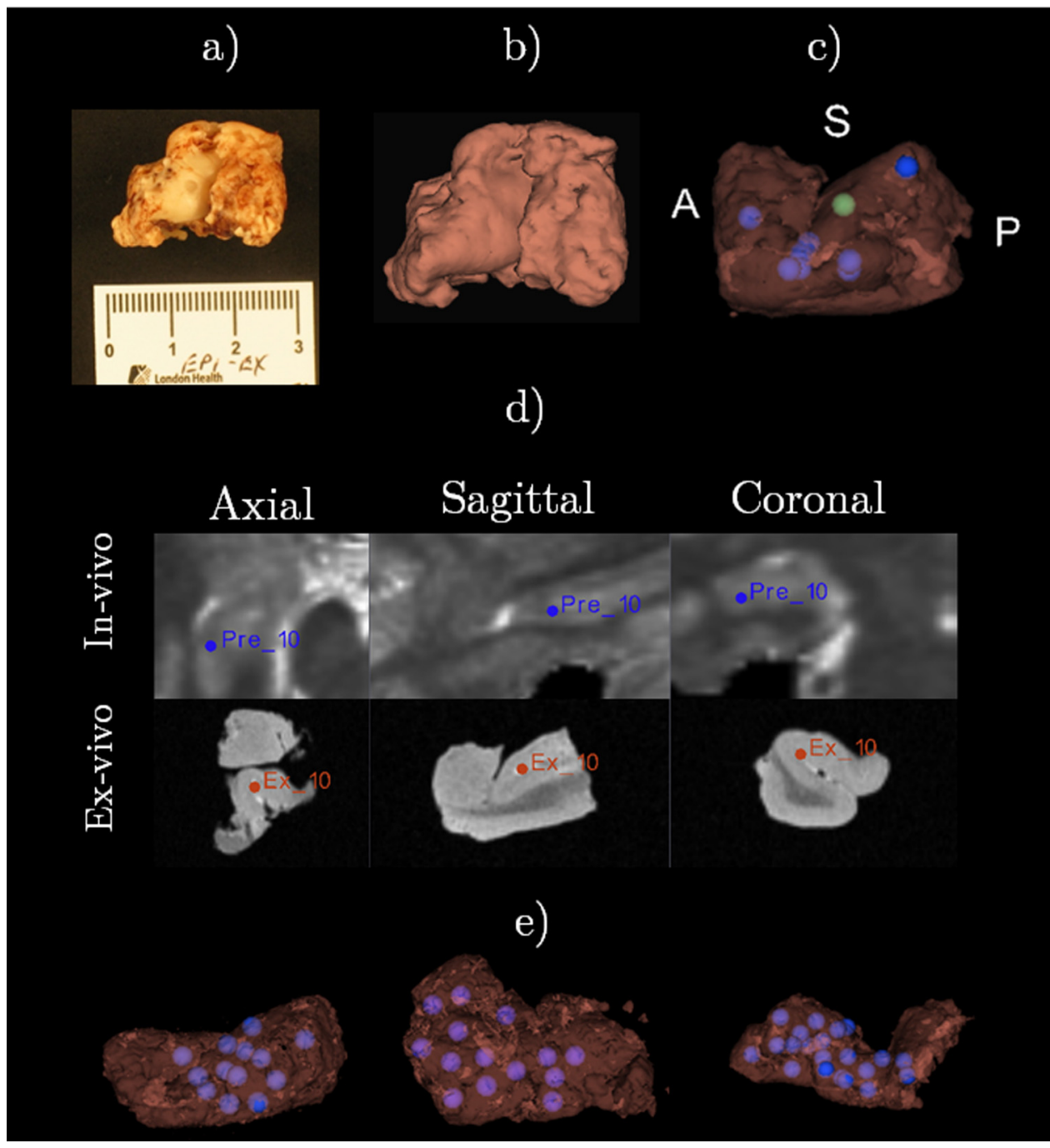

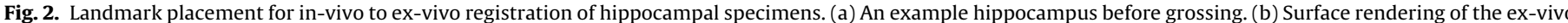

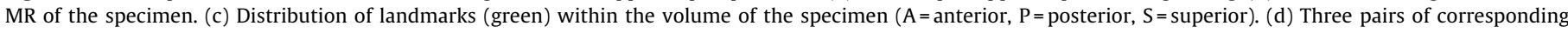

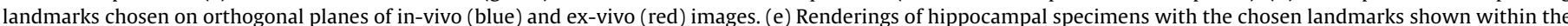
volume to demonstrate their distribution. (For interpretation of the references to colour in this figure legend, the reader is referred to the web version of this article.)

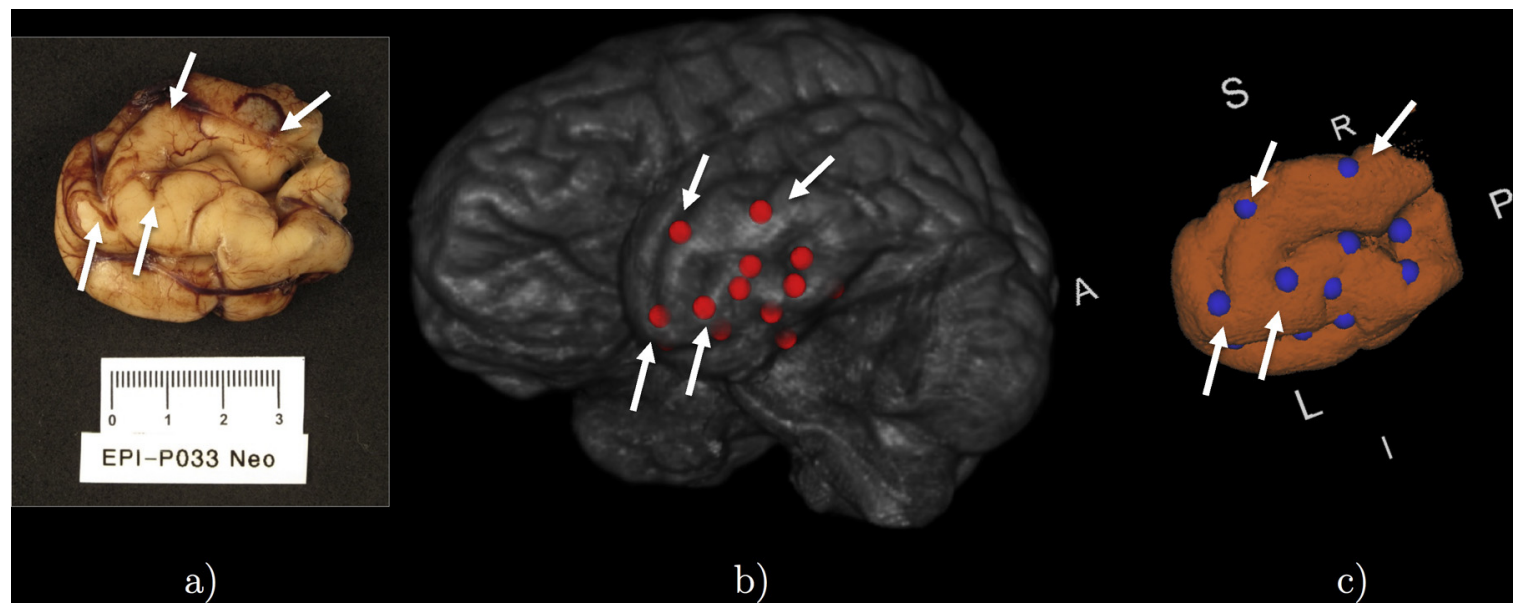

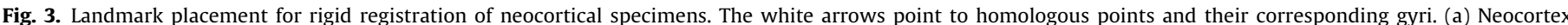

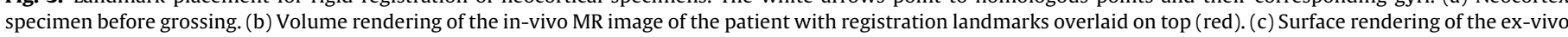

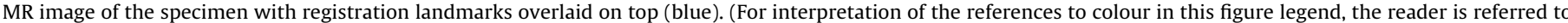
the web version of this article.) 
Table 2

Summary of Dice similarity metric coefficient and root mean squared error (RMSE) for both specimen and tissue types (neocortex \& hippocampus, GM \& WM).

\begin{tabular}{|c|c|c|c|c|}
\hline & \multicolumn{4}{|c|}{ In-vivo to ex-vivo registration error } \\
\hline & \multicolumn{2}{|l|}{ Neocortex } & \multicolumn{2}{|l|}{ Hippocampus } \\
\hline & GM & WM & GM & WM \\
\hline Dice & $0.8750 \pm 0.0099$ & $0.8514 \pm 0.0377$ & $0.8544 \pm 0.0271$ & $0.8380 \pm 0.0390$ \\
\hline RMSE (mm) & $0.3350 \pm 0.1175$ & $0.4048 \pm 0.2387$ & $0.3379 \pm 0.1750$ & $0.3752 \pm 0.2963$ \\
\hline
\end{tabular}

of repeated localizations of the same landmark by the same rater (Fitzpatrick et al., 1998), or the intra-rater variability, described by (1) below:

$T L E=\sqrt{\frac{1}{J}} \sum_{j=1}^{J} \frac{1}{k-1} \sum_{k=1}^{K}\left\|P_{j, k}-\frac{1}{K} \sum_{k=1}^{K} P_{j, k}\right\|^{2}$

where $P j, k$ is the $k$ th localization of the $j$ th landmark. A total of three localization $(K=3)$ of fifteen landmarks $(J=15)$ was performed.

In addition to the landmark-based validation of registration errors, we performed region based by employing the Dice similarity coefficient, defined as: $\mathrm{DSC}=2\left(R_{A} \cap R_{B}\right) /\left(R_{A}+R_{B}\right)$ between delineated regions of interests (ROIs) on the histology and MRI, to further validate the registration accuracy. The cortical gray matter crown and adjacent white matter were chosen as ROIs for neocortical specimens, whereas the entorhinal cortex and combined cornu Ammonis (CA) subfields were chosen as targets for hippocampal specimens. Root mean square error (RMSE) was computed as well for the chosen ROIs in all the specimens. The two dimensional Dice coefficient and RMSE results are summarized in Table 2.

\section{Results}

Our registration protocol produced a mean TRE of $1.46 \pm 0.30 \mathrm{~mm}$ and $1.35 \pm 0.11 \mathrm{~mm}$ for rigid and non-rigid registrations of neocortical specimens respectively, as shown in Fig. 4. Similarly, we computed a TRE of $1.71 \pm 0.36 \mathrm{~mm}$ and $1.41 \pm 0.33 \mathrm{~mm}$ for rigid and non-rigid registrations of hippocampal specimens respectively. The mean landmark localization error was $0.23 \mathrm{~mm}$, which is small relative to an isotropic $1 \mathrm{~mm}$ voxel size of our T1 maps, $I_{\text {in }}$, while the TRE for our repeatability experiment of two independent landmark sets varied by $7 \%$ and $11 \%$ for rigid and non-rigid registrations respectively. The Wilcoxon

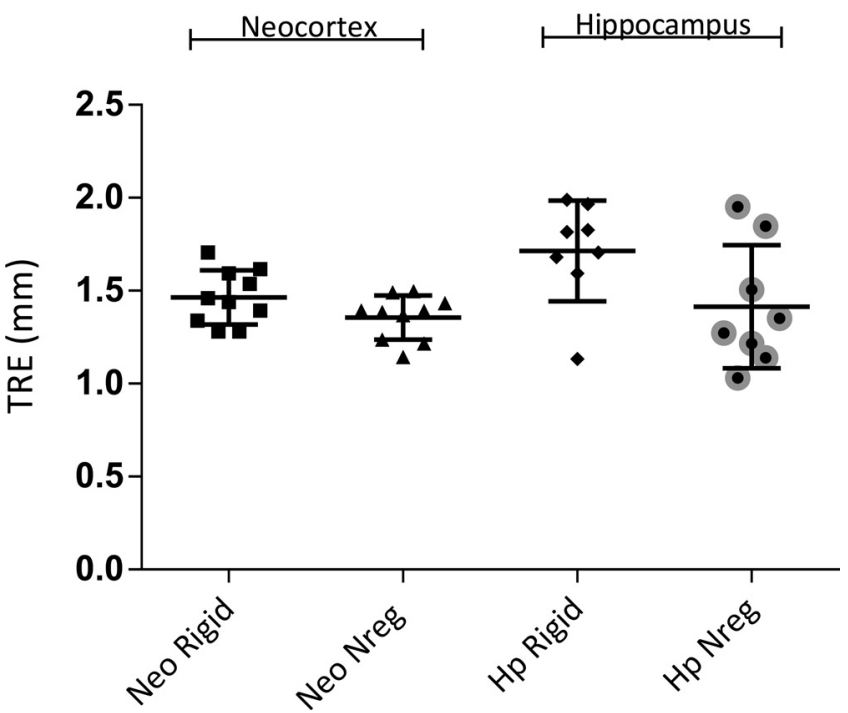

Fig. 4. Boxplots with standard deviation of Hippocampal and Neocortical registration target registration errors at rigid and non-rigid registration stages. matched-pairs signed-rank test confirmed that the deformable registration significantly improved the registration accuracy for the neocortex $(P$ neo $=0.0019,95 \% \mathrm{CI}$ of difference $[-0.20,-0.027])$ and the hippocampus $(\mathrm{Php}=0.0011,95 \% \mathrm{CI}$ of difference $[-0.54$, $-0.038]$ ). The results of the landmark-based rigid and non-rigid registrations for the hippocampi are depicted on an example specimen in Fig. 5. Purple (Fig. 5(b)) and red (Fig. 5(c)) represent rigidly and non-rigidly registered hippocampi respectively. The white arrows in the figure show areas were deformable registration outperformed rigid registration. The last row (Fig. 5(f)) depicts the difference in shape and volume between the hippocampus specimen after rigid transformation and deformable warping. Fig. 6 shows a comparison between the results of the rigid landmarkbased and deformable image-based neocortical registration on an example specimen. As with the hippocampus, the white arrows highlight regions were the non-rigid registration produced a more optimal fit between the images. Table 2 summarizes the region and distance-based validation results for both specimen types and within both brain tissue types, gray matter (GM) and white matter (WM).

Our complete registration pipeline, allows in-vivo MR images to be registered to histology of surgically resected specimens, and brings both modalities to an intermediate reference space, based on ex-vivo MR images. After generating the deformation fields that bring pre-operative images to the ex-vivo space, we warped all the pre-operative scans acquired in the $3 \mathrm{~T}$ MRI scanning session, as well as those acquired using the $7 \mathrm{~T}$ scanner if available. Fig. 7 presents $7 \mathrm{~T}, 3 \mathrm{~T}$, as well as clinical $1.5 \mathrm{~T}$ scans from a single subject registered to the excised neocortical sample. Registered histological slices of a coronal section of the neocortex are also shown. The figure highlights the effect of signal-to-noise (SNR) and image resolution on the quality of registration. It also highlights the potential of warping quantitative $\mathrm{T} 1$ and $\mathrm{T} 2$, as well as diffusion maps to histology for use in region of interest (ROI) or voxel-based analysis. A warped, reconstructed 3D stack of consecutive H\&E stained slices of a neocortical specimen is shown registered to the ex-vivo MRI in Fig. 8(c). This figure highlights as well our 2D affine registration between H\&E and other IHC stained sections ( $\mathrm{f}-\mathrm{i}$ ).

\section{Discussion}

Our in-vivo to ex-vivo MR registration protocol resulted in a target registration error (TRE) of $1.35 \pm 0.11 \mathrm{~mm}$ and $1.41 \pm 0.33 \mathrm{~mm}$ for neocortical and hippocampal specimens respectively. We have previously demonstrated (Goubran et al., 2013) that the errors for the intermediate ex-vivo to histology registration were $0.98 \pm 0.60 \mathrm{~mm}$ and $0.76 \pm 0.66 \mathrm{~mm}$ for neocortical and hippocampal specimens respectively, resulting in a cumulative total error close to $2.33 \mathrm{~mm}$ and $2.17 \mathrm{~mm}$ for the in-vivo to histology registration. Table 3 presents a summary of the studies reporting algorithms for in-vivo MRI to histology of the brain in the last 15 years. Performing voxel-based registration allows for spatially local comparison of MRI and histology, and the scale of this analysis is dependent on the achievable registration accuracy. Many previous studies in MRI and histology registration (see Table 3) did not report accuracy or TRE (Dauguet et al., 2007; Malandain et al., 


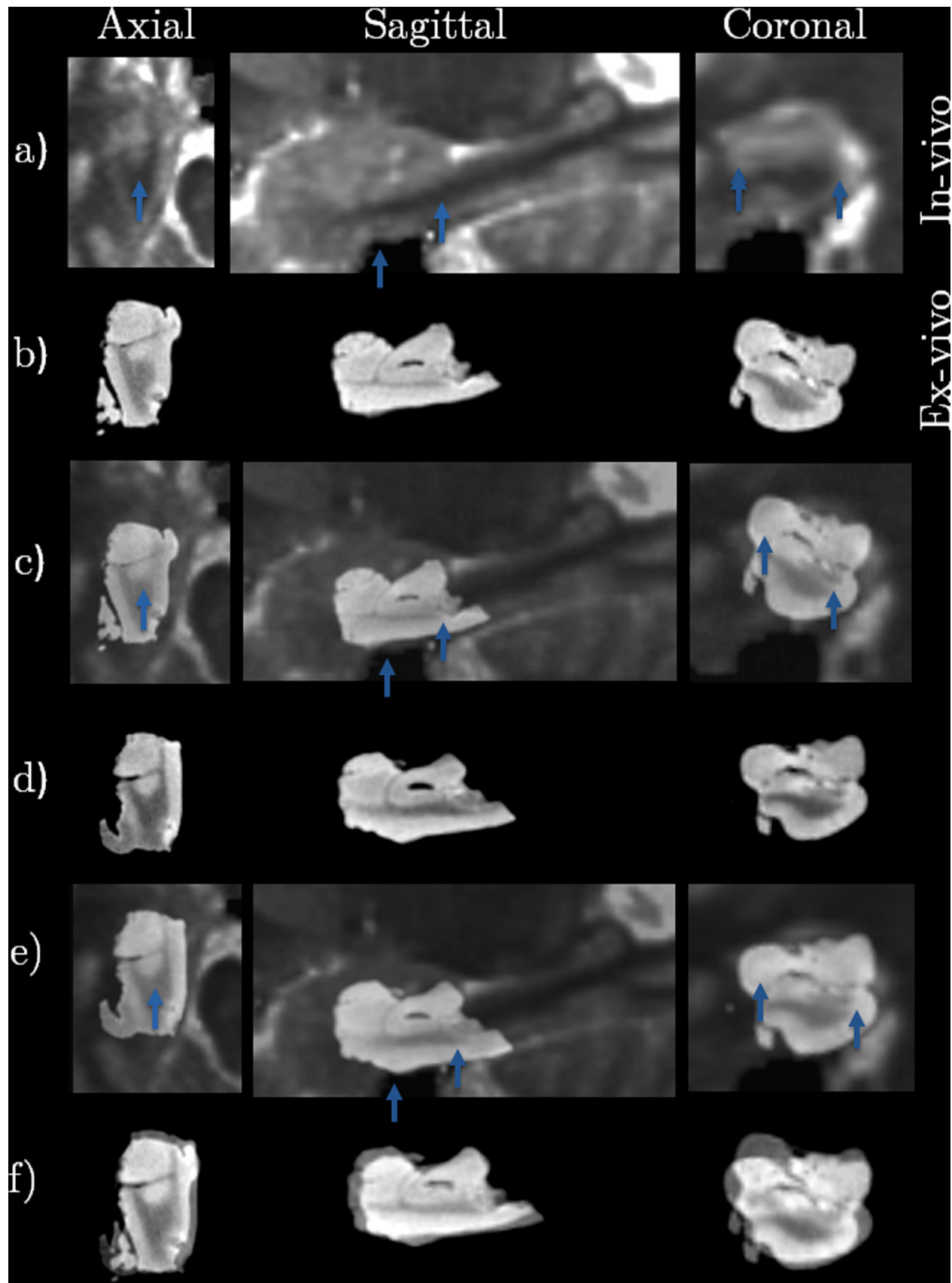

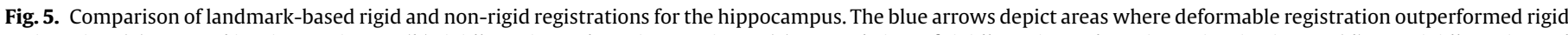

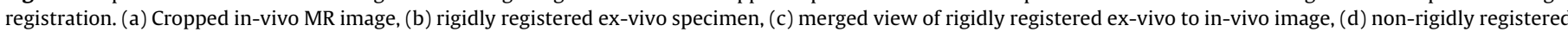

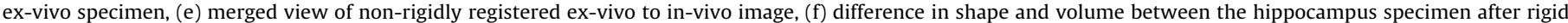
transformation and deformable warping. (For interpretation of the references to colour in this figure legend, the reader is referred to the web version of this article.)

2004; Meyer et al., 2006; Lebenberg et al., 2010; Schormann et al., 1995; Yelnik et al., 2007; Osechinskiy and Kruggel, 2011; Bardinet et al., 2002), and furthermore many previous studies included evaluation on only one dataset (Malandain et al., 2004; Choe et al., 2011; Meyer et al., 2006; Schormann et al., 1995; Kim et al., 2000; Yelnik et al., 2007; Osechinskiy and Kruggel, 2011; Bardinet et al., 2002; Lazebnik et al., 2003). Of the studies that did report accuracy on more than one dataset, TRE ranged from sub-millimeter (Ceritoglu et al., 2010; Jacobs et al., 1999; Yang et al., 2012) to 3-5 mm (Liu et al., 2012; Singh et al., 2008). Techniques that reported sub-millimeter TRE were applied on either whole brain sections of rodents or serially sectioned histology of primates, thus the smaller scale of anatomy and lack of variable resection boundaries can explain the lower TRE relative to our method. For a more relevant comparison (Singh et al., 2008), performed registration of human in-vivo and post-mortem whole brain specimens and reported a TRE of $5.1 \mathrm{~mm}$. The only existing work that dealt with resected temporal lobe specimens was (Eriksson et al., 2005), however they only aimed to find corresponding slices between MRI and histology, and reported inter- and intra-observer variability $(<2 \mathrm{~mm})$ instead of an accuracy measure. This work builds upon these efforts and provides a means to perform voxel-based MRI and histology studies in both neocortical and hippocampal specimens.

Visual comparisons between rigid and deformable registrations results highlight the high specimen deformations that occur during surgery, specifically for small hippocampal specimens as shown in Fig. 5(f). Moreover, the statistically significant improvement of 


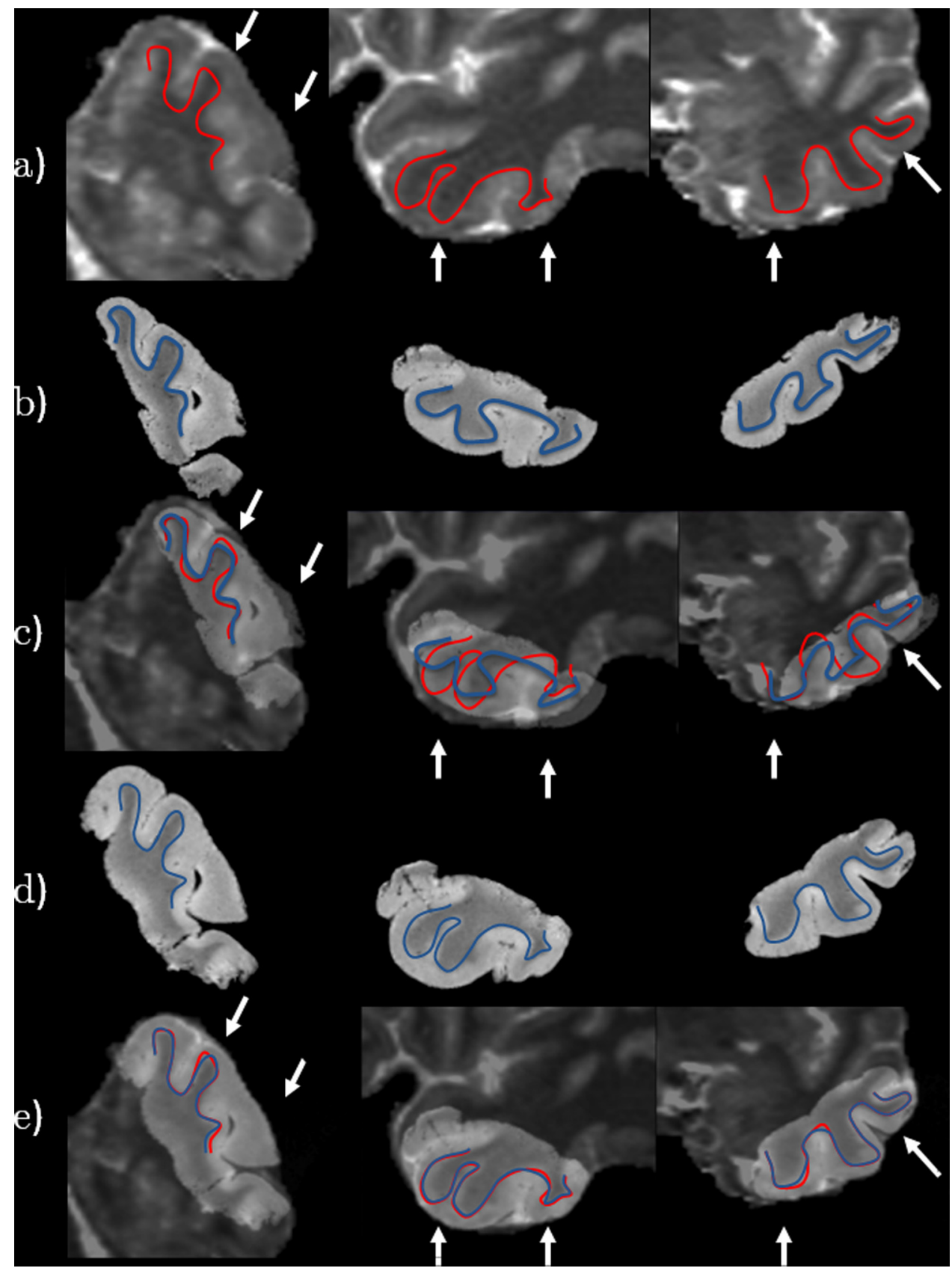

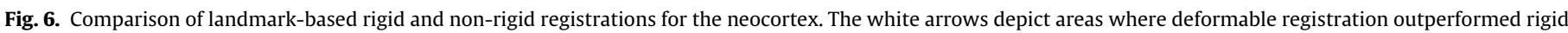

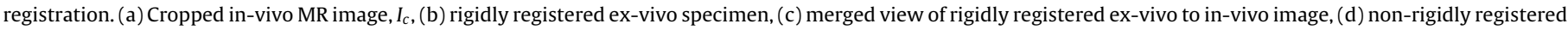
ex-vivo specimen, (e) merged view of non-rigidly registered ex-vivo to in-vivo image.

registration accuracy by non-rigid registration demonstrates the need for a free-form deformable mapping within the registration protocol for a good fit between in-vivo MRI and ex-vivo specimens. These results also demonstrate the pitfall of only relying on rigid or piece-wise rigid registration algorithms, including landmarkbased, for warping hippocampal specimens or pathology to in-vivo space, due to the drastic change in shape and coherence that occurs to the hippocampus after separation from adjacent neighbouring tissue and other support elements (CSF, blood volume). We chose a user-guided landmark-based approach to account for the hippocampal deformations since there is a great deal of variability in how much of the anterior hippocampus and the proximal mesial structures is retrieved from case to case, in part due to the fact that some of the tissue is lost through aspiration. This variability, along with, the smaller size of samples of the hippocampus and histology breakage result in the increased variability observed in TRE of hippocampal specimens as compared to neocortical cases. The application tackled in this manuscript is challenging due to the variable resection boundary, and hence variety of specimen shapes and sizes presented as described earlier, which complicates the implementation of a fully automated reliable non-rigid algorithm. Although, manual landmark placement can be time consuming and userdependent, user anatomical expertise can enforce registration constrains with placed landmarks when similarity metrics fail to find sufficient correspondences between both modalities. We have also shown that our landmark registration protocols are reproducible across different attempts using two independent sets for the same hippocampal specimens. An implementation of a fully automated deformable registration algorithm based on image features would have decreased the amount of time required for performing the 


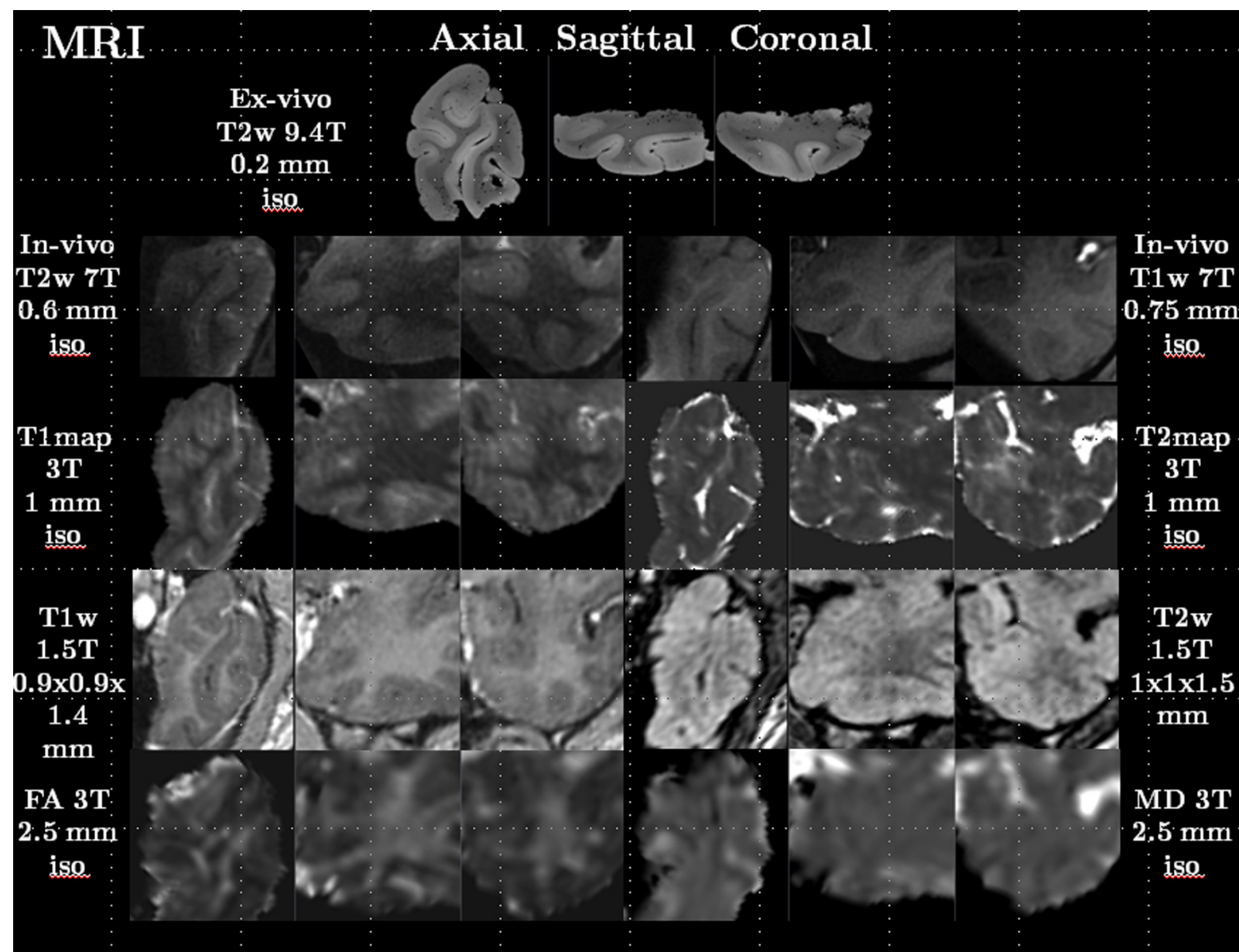

\section{HISTOLOGY}

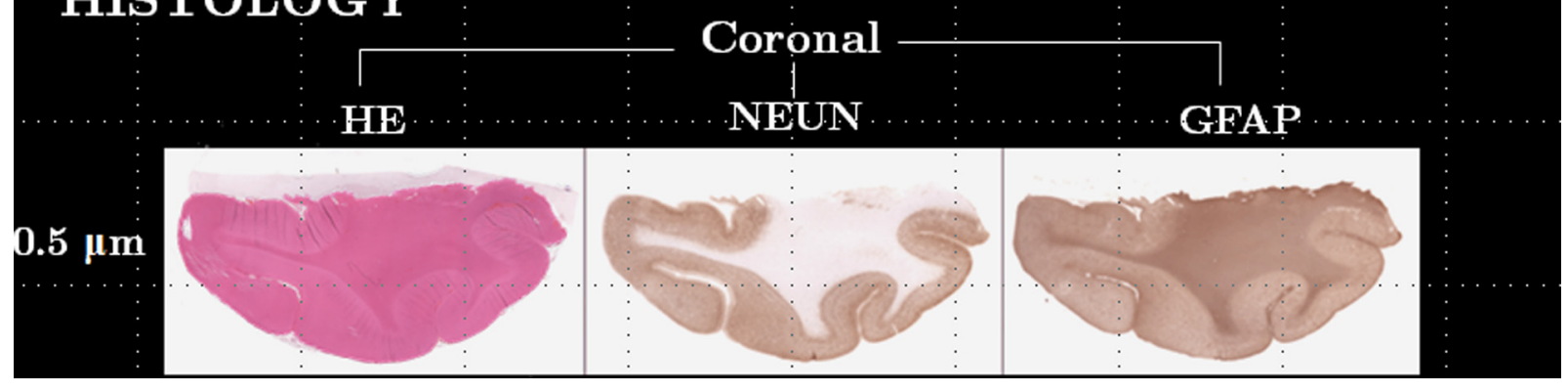

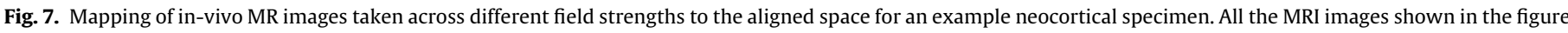

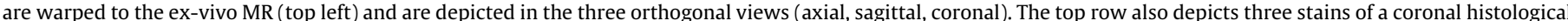

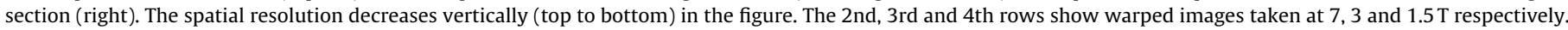

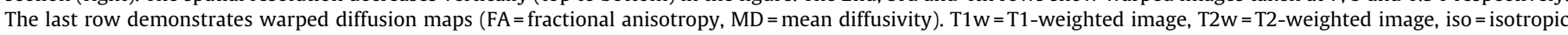
resolution.

registration tasks; however, it may have not improved the registration accuracy specifically for smaller highly deformed specimens. In addition, applying the similarity transform for hippocampal cases before choosing a second set of landmarks for non-rigid registration helped identify landmarks more readily since the anatomy was better aligned at this stage and thus the search space was restricted.

Many studies correlating MRI and histology have been done without the use of computational methods for 3D image registration (Eriksson et al., 2005; Lockwood-Estrin et al., 2012; Garbelli et al., 2012). These rely on visual matching of anatomy or lesions, usually on a slice by slice basis, and are suitable if the tissue of interest (lesion, or anatomy) can be reliably identified in both modalities. However, this is difficult in cases where the visibility or boundaries of the lesion in MRI are different than in histology, or if there is no apparent lesion (as is the case in paradoxical TLE). It is in these cases where MRI-histology registration is crucial, since allows for quantitative investigation of imaging correlates in these lesions that can ultimately improve MRI-based detection and delineation. Another drawback of visual matching is that it becomes more challenging to find corresponding slices when there are 3D deformations present, as the anatomy in a histology slice may not be fully present in a single MRI slice, even if obliquely resampled. Registration methods that can deal with and compensate 3D deformations take the guesswork out of the inherently 2-dimensional visual matching task by using the salient features in the images to define the 3D transformations. Many registration protocols, including ours, also employ specialized tissue handling and slicing protocols designed to minimize deformations and maximize consistency; 


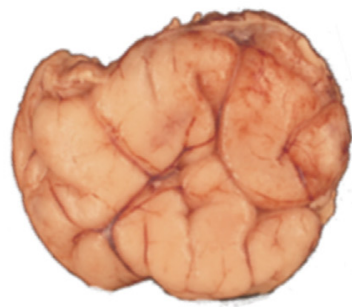

a)

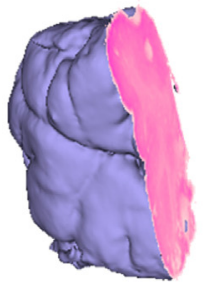

d)

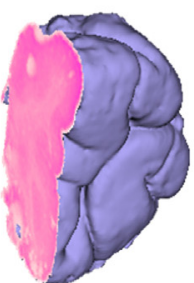

(1)

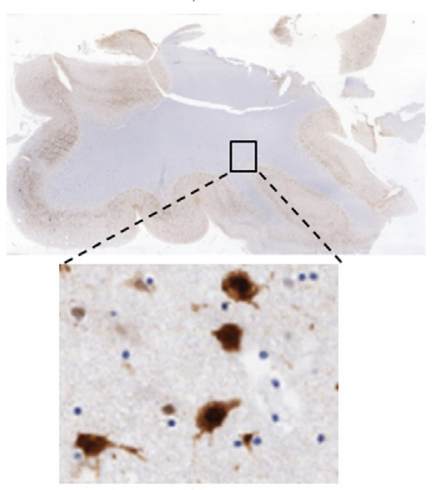

g)

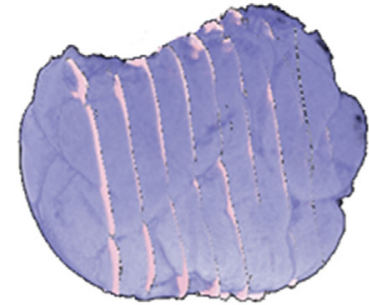

b)

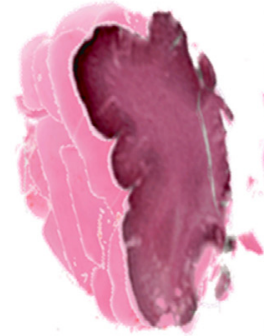

e)

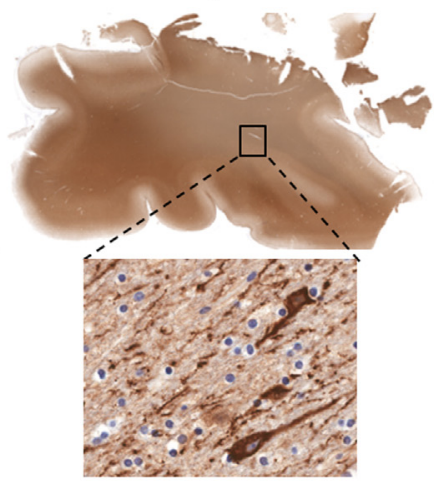

h)

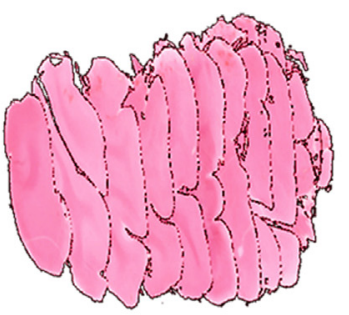

c)

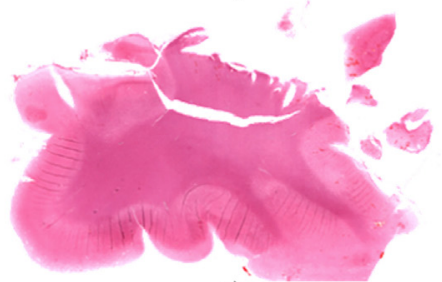

f)

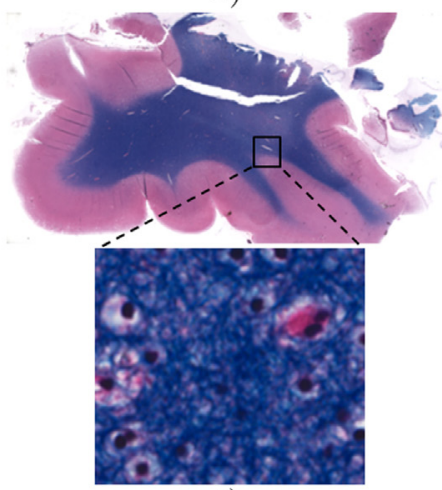

i)

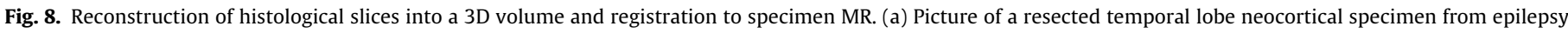

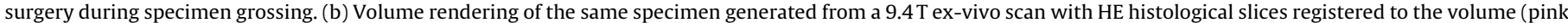

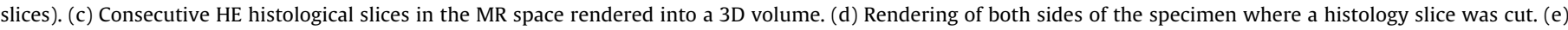

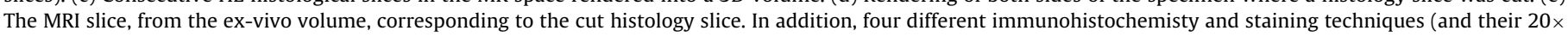

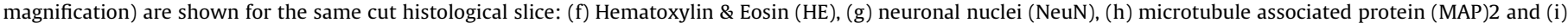
luxol fast blue (LFB). (For interpretation of the references to colour in this figure legend, the reader is referred to the web version of this article.)

these are helpful in many cases but do not preclude the use of further image-based or landmark-based registration to obtain greater accuracy.

We have presented and evaluated a detailed protocol for relating in-vivo MRI and histology, however, a true test of its generalizability would be to implement and validate this protocol in another clinical research setting. There are several limitations which could make translation of this technique difficult. For one, this protocol requires close co-operation with both neurosurgery and pathology departments. En-bloc resections are not always

Table 3

Overview of the studies reporting algorithms for in-vivo MRI to histology of the brain in the last 15 years.

\begin{tabular}{|c|c|c|c|c|c|c|c|c|c|}
\hline Study & Reg features & Trans model & Dim. & Brain section & Mammal & $N$ & Interm. Ref. & Spacing $(\mathrm{mm})$ & TRE $(\mathrm{mm})$ \\
\hline Schormann et al. (1995) & Images & Linear + elastic & $2 D+3 D$ & Whole & PM human & 1 & Block+ ex MRI & 1.8 & $\mathrm{~N} / \mathrm{A}$ \\
\hline Jacobs et al. (1999) & Surfaces + contours & TPS & $3 \mathrm{D}$ & Whole & Rodent & 15 & No & 1 & 0.71 \\
\hline Kim et al. (2000) & Images + landmarks & TPS & $2 \mathrm{D}$ & Whole & Rodent & 1 & Block & 0.02 & $3 \mathrm{px}(2 \mathrm{D})$ \\
\hline Bardinet et al. (2002) & Images & Affine & $2 D+3 D$ & Hemi & PM human & 1 & Block & 0.7 & $\mathrm{~N} / \mathrm{A}$ \\
\hline Lazebnik et al. (2003) & Landmarks & Rigid & $2 D+3 D$ & Whole & Rabbit & 1 & No & 3 & 0.52 \\
\hline Malandain et al. (2004) & Images & Affine & $2 D+3 D$ & Hemi & Primate & 1 & No & 0.04 & $\mathrm{~N} / \mathrm{A}$ \\
\hline Meyer et al. (2006) & User init + images & TPS & 2D (one slice) & Whole & Rodent & 1 & Block+ ex MRI & - & $\mathrm{N} / \mathrm{A}$ \\
\hline Yelnik et al. (2007) & Contours/Images & Affine & $2 \mathrm{D}+3 \mathrm{D}$ & Hemi & PM human & 1 & Block & 0.7 & $\mathrm{~N} / \mathrm{A}$ \\
\hline Dauguet et al. (2007) & Images & Elastic & $2 D+3 D$ & Whole & PM human & 2 & Block & 0.72 & $\mathrm{~N} / \mathrm{A}$ \\
\hline Singh et al. (2008) & Images & Elastic & 3D (4 slices) & Whole & PM human & 11 & Block & 0.03 & 5.1 \\
\hline Lebenberg et al. (2010) & Images & Affine + elastic & $2 \mathrm{D}+3 \mathrm{D}$ & Hemi & Rodent & 7 & Block & 0.08 & $\mathrm{~N} / \mathrm{A}$ \\
\hline Ceritoglu et al. (2010) & Contours/Images & LDDMM & $2 D+3 D$ & Hemi & Primate & 9 & No & 0.8 & 0.39 \\
\hline Osechinskiy and Kruggel (2011) & Landmarks & TPS & $3 \mathrm{D}$ & Hemi & PM human & 1 & No & 150 & $\mathrm{~N} / \mathrm{A}$ \\
\hline Choe et al. (2011) & Landmarks & TPS & $2 \mathrm{D}+3 \mathrm{D}$ & Whole & Primate & 1 & Block & 0.5 & 0.32 \\
\hline Yang et al. (2012) & Contours + images & Rigid + B-spline & $2 D+3 D$ & Whole & Rodent & 4 & No & 0.04 & 0.27 \\
\hline Liu et al. (2012) & Landmarks & TPS & $3 \mathrm{D}$ & Whole & Rodent & 5 & No & 0.04 & 3 \\
\hline
\end{tabular}

TPS, thin plate spline; LDDMM, large deformation diffeomorphic metric mapping. 
performed for temporal lobectomies, and the use of ultrasonic aspirators results in fragmented tissue that is difficult or impossible to deal with in histology correlation studies. A final issue is the reliance on sophisticated imaging resources, such as high-field and ultra-high field magnets for pre-operative and ex-vivo imaging. Despite these limitations, the deployment of our protocol in our unique clinical and research environment allows us to investigate specific clinical questions that can significantly influence our understanding and treatment of temporal lobe epilepsy. The histological basis of focal alterations of relaxometry and diffusion in epilepsy, both proximal and distal to the seizure focus, are important questions that have yet to be resolved (Rugg-Gunn et al., 2002; Jackson et al., 1993) and could also impact the clinical use of such sequences in assessing laterality or localization of epileptogenicity. Our future work involves a registration-based correlation of MRI and histology whereby spatial clusters of abnormal T1, T2, fractional anisotropy, or mean diffusivity, could be compared against histological measures such as neuronal density, gliosis, and myelination. The investigation of mesial temporal sclerosis (MTS) and its sub-types is another area of research which may impact clinical treatment and prediction of surgical outcomes (Blumcke et al., 2012). Our registration of hippocampal specimens is a critical step in the exploration of imaging and histology correlates in sub-regions of the hippocampus and could lead to better pre-operative assessment of hippocampal sub-types and perhaps greater sensitivity to subtle changes in early stages of MTS.

In conclusion, we have implemented and validated a protocol for registration of in-vivo to ex-vivo brain specimen MRI, allowing for the first time a dense correspondence of in-vivo MR with temporal lobe histology. Image registration of surgically resected brain specimens is a unique application which presents a number of technical challenges and that have not been fully addressed in previous literature. The registration accuracy reported is within an acceptable range and allows for the spatially local and quantitative assessment of pathological correlates in MRI by the fusion of information from both modalities.

\section{Acknowledgments}

The authors would like to thank Dr. Seyed Mirsattari, Dr. David Steven and Robert Mayer for their assistance and support throughout the study. This project is funded by the Canadian Institute of Health Research (CIHR) grant MOP 184807 and Canada Foundation for Innovation (CFI) grant 20994. M.G. is supported by the NSERC Create Grant CAMI award at Western University. A.K. is supported by a post-doctoral fellowship from the Canadian Institute of Health Research (CIHR).

\section{References}

Amunts K, Lepage C, Borgeat L, Mohlberg H, Dickscheid T, Rousseau MÉ, et al. BigBrain: an ultrahigh-resolution 3D human brain model. Science 2013;340(6139):1472-5

Bardinet E, Ourselin S, Dormont D, Malandain G, Tandé D, Parain K, et al. Co-registration of histological, optical and MR data of the human brain. Medical Image Computing and Computer-Assisted Intervention-MICCAI 2002;2488:548-55.

Bernasconi A, Bernasconi N, Caramanos Z, Reutens DC, Andermann F, Dubeau F, et al. T2 relaxometry can lateralize mesial temporal lobe epilepsy in patients with normal MRI. Neuroimage 2000;12(6):739-46.

Bernasconi N, Duchesne S, Janke A, Lerch J, Collins DL, Bernasconi A. Whole-brain voxel-based statistical analysis of gray matter and white matter in temporal lobe epilepsy. Neuroimage 2004;23(2):717-23.

Bernhardt BC, Worsley KJ, Kim H, Evans AC, Bernasconi A, Bernasconi N. Longitudinal and cross-sectional analysis of atrophy in pharmacoresistant temporal lobe epilepsy. Neurology 2009;72(20):1747-54.

Blumcke I, Coras R, Miyata H, Ozkara C. Defining clinico-neuropathological subtypes of mesial temporal lobe epilepsy with hippocampal sclerosis. Brain Pathol 2012;22(3):402-11.
Breen MS, Lancaster TL, Wilson DL. Correcting spatial distortion in histologica images. Comput Med Imaging Graph 2005;29(6):405-17.

Ceritoglu C, Wang L, Selemon LD, Csernansky JG, Miller MI, Ratnanather JT. Large deformation diffeomorphic metric mapping registration of reconstructed 3D histological section images and in vivo MR images. Front Hum Neurosci 2010;4:43.

Chappelow J, Bloch BN, Rofsky N, Genega E, Lenkinski R, DeWolf W, Madabhushi A Elastic registration of multimodal prostate MRI and histology via multiattribute combined mutual information. Med Phys 2011;38(4):2005-18.

Choe AS, Gao Y, Li X, Compton KB, Stepniewska I, Anderson AW. Accuracy of image registration between MRI and light microscopy in the ex vivo brain. Magn Reson Imaging 2011;29(5):683-92.

Dauguet J, Delzescaux T, Condé F, Mangin JF, Ayache N, Hantraye P, Frouin V. Three-dimensional reconstruction of stained histological slices and 3D nonlinear registration with in-vivo MRI for whole baboon brain. J Neurosci Methods 2007;164(1):191-204.

de Tisi J, Bell GS, Peacock JL, McEvoy AW, Harkness WF, Sander JW, Duncan JS. The long-term outcome of adult epilepsy surgery, patterns of seizure remission, and relapse: a cohort study. Lancet 2011;378(9800):1388-95.

Deoni SCL. High-resolution T1 mapping of the brain at $3 \mathrm{~T}$ with driven equilibrium single pulse observation of T1 with high-speed incorporation of RF field inhomogeneities (DESPOT1-HIFI). J Magn Reson Imaging 2007;26(4): 1106-11.

Deoni SCL. Transverse relaxation time (T2) mapping in the brain with off-resonance correction using phase-cycled steady-state free precession imaging. J Magn Reson Imaging 2009;30(2):411-7.

Engel J. Etiology as a risk factor for medically refractory epilepsy: a case for early surgical intervention. Neurology 1998;51(5):1243-4.

Engel J, Levesque MF, Shields WD. Surgical treatment of the epilepsies: presurgical evaluation. Clin Neurosurg 1992;38:514-34

Eriksson SH, Free SL, Thom M, Harkness W, Sisodiya SM, Duncan JS. Reliable registration of preoperative MRI with histopathology after temporal lobe resections. Epilepsia 2005;46(10):1646-53.

Eriksson SH, Free SL, Thom M, Martinian L, Symms MR, Salmenpera TM, et al. Correlation of quantitative MRI and neuropathology in epilepsy surgical resection specimens - T2 correlates with neuronal tissue in gray matter. Neuroimage 2007;37(1):48-55.

Fitzpatrick JM, West JB, Maurer CR. Predicting error in rigid-body point-based registration. IEEE Trans Med Imaging 1998;17(5):694-702.

Garbelli R, Milesi G, Medici V, Villani F, Didato G, Deleo F, et al. Blurring in patients with temporal lobe epilepsy: clinical, high-field imaging and ultrastructura study. Brain 2012;135(8):2337-49.

Goubran M, Crukley C, de Ribaupierre S, Peters TM, Khan AR. Image registration of ex-vivo MRI to sparsely sectioned histology of hippocampal and neocortical temporal lobe specimens. Neuroimage 2013;83:770-81.

Howe KL, Dimitri D, Heyn C, Kiehl TR, Mikulis D, Valiante T. Histologically confirmed hippocampal structural features revealed by 3 T MR imaging: potentia to increase diagnostic specificity of mesial temporal sclerosis. AJNR Am J Neuroradiol 2010;31(9):1682-9.

Humm JL, Ballon D, Hu YC, Ruan S, Chui C, Tulipano PK, et al. A stereotactic method for the three-dimensional registration of multi-modality biologic images in animals: NMR, PET, histology, and autoradiography. Med Phys 2003;30(9):2303-14.

Jackson G, Connelly A, Duncan J, Grunewald R, Gadian D. Detection of hippocampal pathology in intractable partial epilepsy increased sensitivity with quantitative magnetic resonance T2 relaxometry. Neurology 1993;43(9):1793.

Jacobs MA, Windham JP, Soltanian-Zadeh H, Peck DJ, Knight RA. Registration and warping of magnetic resonance images to histological sections. Med Phys 1999;26(8):1568-78.

Kim TS, Singh M, Sungkarat W, Zarow C, Chui H. Automatic registration of postmortem brain slices to MRI reference volume. Nuclear Sci IEEE Trans 2000;47(4):1607-13.

Lazebnik RS, Lancaster TL, Breen MS, Lewin JS, Wilson DL. Volume registration using needle paths and point landmarks for evaluation of interventional MRI treatments. IEEE Trans Med Imaging 2003;22(5):653-60.

Lebenberg J, Hérard AS, Dubois A, Dauguet J, Frouin V, Dhenain M, et al. Validation of MRI-based 3D digital atlas registration with histological and autoradiographic volumes: an anatomofunctional transgenic mouse brain imaging study. Neuroimage 2010;51(3):1037-46.

Liu Y, Sajja BR, Uberti MG, Gendelman HE, Kielian T, Boska MD. Landmark optimization using local curvature for point-based nonlinear rodent brain image registration. Int J Biomed Imaging 2012;2012:1.

Lockwood-Estrin G, Thom M, Focke NK, Symms MR, Martinian L, Sisodiya SM, et al Correlating $3 \mathrm{~T}$ MRI and histopathology in patients undergoing epilepsy surgery. J Neurosci Methods 2012;205(1):182-9.

Malandain G, Bardinet E, Nelissen K, Vanduffel W. Fusion of autoradiographs with an MR volume using 2-D and 3-D linear transformations. Neuroimage 2004;23(1):111-27.

Meyer CR, Moffat BA, Kuszpit K, Bland PL, Chenevert TL, Rehemtulla A, Ross BD. A methodology for registration of a histological slide and in vivo MRI volume based on optimizing mutual information. Mol Imaging 2006;5(1):16-23.

Modat M, Ridgway GR, Taylor ZA, Lehmann M, Barnes J, Hawkes DJ, et al. Fast freeform deformation using graphics processing units. Comput Methods Programs Biomed 2010;98(3):278-84.

Osechinskiy S, Kruggel F. Slice-to-volume nonrigid registration of histological sections to MR images of the human brain. Anat Res Int 2011;2011:1. 
Pinter C, Lasso A, Wang A, Jaffray D, Fichtinger G. Slicer RT: radiation therapy research toolkit for 3D slicer. Med Phys 2012;39:6332-8.

Rueckert D, Sonoda LI, Hayes C, Hill DL, Leach MO, Hawkes DJ. Nonrigid registration using free-form deformations: application to breast MR images. IEEE Trans Med Imaging 1999;18(8):712-21.

Rugg-Gunn FJ, Eriksson SH, Symms MR, Barker GJ, Thom M, Harkness W, Duncan JS. Diffusion tensor imaging in refractory epilepsy. Lancet 2002;359(9319):1748-51.

Schormann T, Dabringhaus A, Zilles K. Statistics of deformations in histology and application to improved alignment with MRI. IEEE Trans Med Imaging 1995:14(1):25-35.

Singh M, Rajagopalan A, Kim TS, Hwang D, Chui H, Zhang XL, et al. Co-registration of in-vivo human MRI brain images to postmortem histological microscopic images. Int J Imaging Syst Technol 2008;18(5-6):325-35.
Sylaja P, Radhakrishnan K, Kesavadas C, Sarma P. Seizure outcome after anterior temporal lobectomy and its predictors in patients with apparent temporal lobe epilepsy and normal MRI. Epilepsia 2004;45(7):803-8.

Ward AD, Crukley C, McKenzie C, Montreuil J, Gibson E, Gomez JA, et al. Registration of in vivo prostate magnetic resonance images to digital histopathology images. Prostate Cancer Imaging Computer-Aided Diagnosis, Prognosis, and Intervention 2010;6367:66-76.

Yang Z, Richards K, Kurniawan ND, Petrou S, Reutens DC. MRI-guided volume reconstruction of mouse brain from histological sections. J Neurosci Methods 2012;211:210-7.

Yelnik J, Bardinet E, Dormont D, Malandain G, Ourselin S, Tandé D, et al. A threedimensional, histological and deformable atlas of the human basal ganglia. I. Atlas construction based on immunohistochemical and MRI data. Neuroimage 2007; 34(2):618-38. 